\title{
Caso Vera: Intervenções do Psicoterapeuta e Aliança Terapêutica
}

\author{
Evandro Morais Peixoto ${ }^{1}$ \\ Programa de Pós-Graduação em Psicologia como Profissão e Ciência da Pontifícia \\ Universidade Católica de Campinas, Campinas, SP, Brasil \\ Giovanna Corte Honda \\ Universidade Nove de Julho, São Paulo, SP, Brasil \\ Maria Leonor Espinosa Enéas \\ Centro de Ciências Biológicas e da Saúde da Universidade Presbiteriana Mackenzie, \\ São Paulo, SP, Brasil \\ Glaucia Mitsuko Ataka da Rocha \\ Estudos Avançados - Centro Universitário São Camilo, São Paulo, SP, Brasil \\ Faculdade de Medicina de Marília, Marilia, SP, Brasil \\ Instituto de Psicologia da Universidade de São Paulo, São Paulo, SP, Brasil \\ Sonia Maria da Silva \\ Universidade Guarulhos, Guarulhos, SP, Brasil \\ Daniela Wiethaeuper \\ Département de Psychologie, Université du Québec à Trois-Rivières, \\ Trois-Rivières, Québec, Canadá
}

\section{Resumo}

Entre os fatores que contribuem para o êxito das psicoterapias estão associados aspectos do psicoterapeuta, do paciente e da qualidade da relação entre ambos. Esta pesquisa objetivou avaliar um processo breve psicodinâmico relacionando dois destes aspectos, as intervenções verbais da psicoterapeuta e a aliança terapêutica estabelecida entre paciente e psicoterapeuta. Empregou-se estudo de caso único e instrumentos clínicos para avaliar as variáveis propostas: Classificação das Intervenções Terapêuticas (IT) e Inventário de Aliança de Trabalho - versão do observador (Working Alliance Inventory Short - observer version - WAI-S-O). Os resultados revelaram que a estratégia interventiva adotada pela psicoterapeuta foi predominantemente expressiva e houve aumento nas médias da aliança terapêutica da fase inicial para as demais. Pode-se perceber, também, que as intervenções foram modeladas segundo as características inerentes às diferentes fases do processo terapêutico, em especial a etapa de término, onde o conflito central de relacionamento da paciente, o medo de ser abandonada, pode ser reeditado e elaborado. Limitações do estudo são apontadas.

Palavras-chave: Mudança em psicoterapia (psicologia), processos psicoterapêuticos, estudo de caso único.

Endereço para correspondência: Rua Liliane Regina, 03, Vila Cretti, Carapicuíba, SP, Brasil 06386-300. Fone: (11) 98089-4423. E-mail: epeixoto_6@hotmail.com

Financiamento: Coordenação de Aperfeiçoamento de Pessoal de Nível Superior (CAPES). 


\title{
Vera Case: Psychotherapist Interventions and Therapeutic Alliance
}

\begin{abstract}
Variables relating to the therapist, the patient and the quality of their relationship are associated with factors that contribute to the success of the psychotherapy. The main goal of this study was to evaluate a brief psychodynamic psychotherapy process and to establish a relation between two of these aspects: the therapist's verbal interventions and the therapeutic alliance. A single-subject case study was used as well as clinical instruments to evaluate the proposed variables: Therapeutic Interventions Classification (TI) and the Working Alliance Inventory Short - Observer version (WAI-S-O). The results pointed to the therapist's intervention strategy, predominantly expressive, and an increase in the average of the therapeutic alliance from the initial phase through the other phases. Moreover, the interventions were also modulated by the inherent characteristics of the different phases of the therapeutic process, in particular the final stage, where the central conflict of the patient relationship, the fear of being abandoned, could be reedited and elaborated. Limitations of the study are indicated.
\end{abstract}

Keywords: Psychotherapy change (psychology), psychotherapeutic processes, single case study.

\section{Caso Vera: El Rol del Psicoterapeuta e la Alianza de Trabajo}

\section{Resumen}

Variables del terapeuta, del paciente y de la cualidad de la relación entre ambos están asociadas con los factores que contribuyen para el éxito de las psicoterapias. Esta investigación tuvo como objetivo evaluar un proceso breve psicodinámico y establecer una relación entre las intervenciones verbales de la psicoterapeuta y la alianza entre psicoterapeuta y paciente. Se utilizó estudio de caso único y instrumentos clínicos para evaluar las variables propuestas: Clasificación de las Intervenciones Terapéuticas (IT); Tema Central de Conflictos en los Relacionamientos (Core Conflictual Relationship Theme - CCRT); y Inventario de la Alianza de Trabajo - versión del observador (Working Alliance Inventory Short - observer version - WAI-S-O). Los resultados demonstran que la estrategia de las intervenciones usadas por la psicoterapeuta, en su mayor parte expresivas, contribuyó para el desarrollo de la alianza terapéutica, mejora del patrón relacional y disminución de los problemas relacionados a la queja. Sin embargo, es posible percibir que las intervenciones también fueron moduladas por las características inherentes a las distintas fases del proceso terapéutico, en particular la etapa de finalización, en que el conflicto central de la relación la paciente, el miedo a ser abandonada, pudo ser vivido y elaborado. Limitaciones del estudio son apuntadas.

Palabras clave: Cambio en psicoterapia (psicología), procesos psicoterapéuticos, estudio de caso único.

Estudos realizados nas últimas quatro décadas indicam que as psicoterapias, independentes de orientação teórica e modalidade de atendimento, contribuem para promoção de mudanças nos pacientes (Prochaska, 1995; Yoshida, 2012). A existência de fatores comuns a todas as abordagens psicoterápicas pode explicar esses achados, à medida que os fatores que contribuem para o êxito das psicoterapias estão associados a aspectos do psicoterapeuta, do paciente e da qualidade da relação entre ambos (Lhullier, Nunes, \& Horta, 2006; Meyer, 2006; Santibáñez Fernandez et al., 2008). Nessa direção, esta pesquisa tem como principal objetivo a avaliação 
de um processo psicoterapêutico breve psicodinâmico relacionando variáveis do terapeuta e da relação entre os dois.

No que se refere aos aspectos do psicoterapeuta, Fiorini (2004) e Gabbard (2006) afirmam ser possível que sua participação no processo seja aferida mediante suas intervenções verbais. As estratégias interventivas, identificadas por meio dos tipos de intervenções que o psicoterapeuta realiza ao longo das sessões, podem influenciar diretamente os resultados do processo (Fiorini, 2004; Gabbard, 2006; Khater, Peixoto, Honda, Enéas, \& Yoshida, 2014). Para Gabbard (2006) e Luborsky (1984), as intervenções na prática das psicoterapias psicodinâmicas podem ser classificadas em um continuum Suportivo-Expressivo. As técnicas de natureza "suportiva" objetivam a manutenção do nível de funcionamento do paciente. São técnicas que demonstram o quanto o psicoterapeuta compreende as situações do paciente, o que aumenta a probabilidade de estabelecimento da aliança terapêutica entre ambos. Já as técnicas de natureza "expressiva" têm como objetivo facilitar a comunicação e compreensão, por parte do paciente, de seus problemas e conflitos inconscientes (Luborsky, 1984). Em se tratando de psicoterapia psicodinâmica é esperado que as intervenções não sejam limitadas a um polo ou outro, mas que haja um equilíbrio ótimo entre umas e outras que possa beneficiar a qualidade da aliança terapêutica (Despland, Roten, Despars, Stigler, \& Perry, 2001).

A relação entre psicoterapeuta e paciente pode ser expressa pela variável aliança terapêutica, também conhecida por aliança de trabalho, aliança de ajuda, vínculo ou laço terapêutico. Para Martin, Garske e Davis (2000) esta variação conceitual é decorrente das diferentes edificações teóricas existentes na psicologia. Contudo, todas as abordagens ressaltam a importância da aliança terapêutica no processo psicoterápico, e entende-se que uma vez estabelecida pode gerar resultados favoráveis ao processo (Falkenström, Granström, \& Homqvist, 2014; Luborsky, 2000; Yoshida \& Enéas, 2013).

Especificamente em relação à influência da qualidade da aliança do terapeuta ou do paciente sobre os resultados do processo, Del
Re, Flückiger, Horvath, Symonds, e Wampold (2012) realizaram uma metanálise e concluíram que a variabilidade na aliança por parte do psicoterapeuta se mostra mais importante sobre os resultados da terapia quando comparada com à do paciente. Sugerem, portanto, que os programas de formação de futuros terapeutas focalizem o desenvolvimento e o fortalecimento de habilidade do terapeuta em estabelecer aliança terapêutica com seus pacientes. Neste sentido, Safran et al. (2014) investigaram o impacto do treinamento focalizado na aliança e levantaram evidências de que este tipo de treinamento tem impacto positivo no processo interpessoal durante as sessões, bem como na capacidade de o terapeuta refletir sobre o relacionamento psicoterapêutico, incorporando sua própria experiência a essa reflexão.

Apesar de haver muitas pesquisas sobre aliança terapêutica, Wiseman e Tishby (2014) destacaram os desafios a serem ainda enfrentados, como por exemplo, o de entender a função do relacionamento entre terapeuta e paciente no processo de mudança, o seu desenvolvimento ao longo das diferentes fases do processo e o tipo de influência que cada um tem sobre a qualidade deste relacionamento.

A partir da compreensão da importância da aliança entre psicoterapeuta e paciente no processo psicoterápico, pesquisadores passaram a reunir esforços para construção de ferramentas capazes de mensurar esse construto e buscar evidências empíricas de relações mais estreitas com resultados da psicoterapia e características de psicoterapeutas e pacientes (Muran \& Barber, 2010). Um dos instrumentos mais utilizados para sua avaliação é o Inventário de Aliança de Trabalho - WAI (Working Alliance Inventory; Bernecker, Levy, \& Ellison, 2014; Horvath \& Greenberg, 1989). O WAI é um instrumento com versões para paciente, terapeuta e observador Busseri e Tyler (2003). De acordo com Prado e Meyer (2004), o instrumento destaca-se por estar entre os que mais aparecem na literatura especializada devido às suas evidências de precisão e validade, possibilidade de ser utilizado no decorrer do processo psicoterapêutico, uma vez que não aborda as sessões em si, mas sim 
o objetivo, a tarefa e a relação entre terapeutapaciente.

Entre as justificativas para realização desta pesquisa, destaca-se que embora alguns estudos tenham enfatizado a interação entre variáveis do terapeuta, como as intervenções psicoterapêuticas, e variáveis do paciente, como o padrão relacional, por exemplo (Slonim, Shefler, Gvirsman, \& Tishby, 2011; Tishby \& Vered, 2011; Yoshida et al., 2009), variáveis de interação entre terapeuta e paciente ainda necessitam de mais estudos. Buscou-se, então, explorar possíveis relações entre as estratégias interventivas adotadas pela psicoterapeuta e a qualidade da aliança terapêutica estabelecida entre paciente e psicoterapeuta.

\section{Método}

O método empregado foi o estudo de caso único, naturalístico, que permite compreender durante a sessão as características de padrões interacionais e comportamentais entre paciente e psicoterapeuta, através do exame intensivo e sistemático do caso (Eells, 2007; Meyer, 2006; Yoshida et al., 2009).

\section{Caso Clínico}

Paciente: mulher, de 23 anos, estudante universitária, encaminhada para psicoterapia por professor de seu curso.

Queixas: Apresentava transtorno alimentar, mais especificamente bulimia. Apesar de demonstrar intenção de eliminar esses sintomas, sentia-se muito desapontada por não obter sucesso nessas tentativas.

Psicoterapia: 11 sessões individuais semanais de cinquenta minutos de duração, realizada em clínica-escola.

Histórico: nascida em uma família de classe média, passava por dificuldades financeiras após o adoecimento e desemprego do pai, o que a deixava muito preocupada. Não trazia lembranças da mãe, visto que esta havia falecido quando ainda era criança de pouca idade, tendo como recordação apenas uma foto, que guardava com muito afeto. Depois que a mãe faleceu, o pai enviou-a para ser cuidada pela avó. Ele iniciou um relacionamento logo após a morte da esposa e a paciente voltou a morar com o pai quando ele casou novamente. A paciente apresentava muitas dificuldades em relacionar-se com sua madrasta e a filha dela. Por fim, relatava com forte carga emocional, o relacionamento com o namorado, que era percebido como um ponto de apoio.

Objetivo do processo terapêtico: desenvolver postura assertiva frente aos relacionamentos, elevando a autoestima da paciente e diminuindo o sentimento de inferioridade, para que deixasse de se submeter às imposições externas.

Terapeuta: mulher, 24 anos de idade, um ano de formação e o mesmo tempo de experiência clínica. O processo psicoterapêutico ocorreu sob supervisão em Psicoterapia Breve de Adultos, realizada por uma das autoras da pesquisa cuja experiência clínica aproximava-se de 25 anos. $\mathrm{O}$ atendimento foi realizado em clínica-escola da universidade em que a pesquisa foi realizada

\section{Instrumentos}

Classificação das Intervenções Terapêuticas (IT) - Classificação adotada por Yoshida, Gatti, Enéas e Coelho-Filho (1997), composta por 14 tipos de intervenções de natureza Expressiva, Neutra ou Suportiva. Todas as intervenções realizadas durante a psicoterapia são analisadas e classificadas de acordo com o sentido evidenciado por meio de cada uma e que faz alusão a alguma das seguintes definições:

1. Interpretação - tornar consciente algo anteriormente inconsciente;

2. Confrontação - identifica algo que o paciente minimiza ou evita;

3. Clarificação - reformulação ou reunião de falas do paciente para torná-las mais coerentes;

4. Assinalamento - mostrar relações entre os dados;

5. Encorajamento a elaborar - pedido de mais informações;

6. Validação empática - demonstra a sintonia empática do terapeuta;

7. Recapitulação - resume pontos essenciais da sessão ou do tratamento; 
8. Conselhos e elogios - recomenda e reforça certas atitudes;

9. Afirmação - comentários sucintos que apoiam comentários e atitudes do paciente;

10. Interrogação - consultar e avaliar a consciência do paciente;

11. Fornecer informações - esclarecer aspectos técnicos desconhecidos do paciente;

12. Intervenções interrompidas - início de intervenção do psicoterapeuta interrompida pelo paciente;

13. Meta intervenção-Objetiva esclarecer o motivo de ter feito alguma outra intervenção naquele momento da sessão ou tratamento;

14. Dar enquadramento - intervenções relativas aos aspectos do enquadre terapêutico.

Essa avaliação possibilita estimar o grau de apoio e de expressividade de todas as intervenções, uma vez que são categorizadas quanto à sua natureza, dentro de um continuum, no qual intervenções direcionadas ao relacionamento ou focalizadas na transferência são mais expressivas (como Interpretação) e intervenções não focalizadas na transferência são mais suportivas (como Conselho e elogio), passando pelas intervenções de natureza Neutra, localizadas no centro do continuum Expressivo-Suportivo. Deste modo, as intervenções n. 1, 2, 3, 4 e 7 são categorizadas como de natureza expressiva, as intervenções de n. 6, 8, e 11 de natureza suportiva e as intervenções de n. 5, 9, 10,13 e 14 são as consideradas neutras. Por fim a intervenção n. 12 não é categorizada, pois se trata de intervenção interrompida. Nesta pesquisa apenas as duas primeiras dimensões (expressiva e suportiva) foram adotadas para fins de avaliação, visto que estas objetivam estimar o grau de expressividade e de apoio, em cada fase do processo.

Inventário de Aliança de Trabalho - Versão do Observador (Working Alliance Inventory Short - Observer Version - WAI-S-O; Tracey \& Kokotovic, 1989) traduzido para o português por Machado e Horvath (1999). O instrumento é composto por doze itens que objetivam a avaliação da Aliança Terapêutica (AT) por observadores externos ao processo terapêutico, agrupados em 3 dimensões: (a) Objetivos - refere-se ao entendimento entre terapeuta e paciente acerca dos objetivos da psicoterapia com a finalidade de promover mudanças; (b) Tarefa - refere-se às atividades desenvolvidas pelo terapeuta e paciente para mobilizar mudanças; e (c) Vínculo refere-se à ligação entre terapeuta e paciente que envolve confiança, respeito e comprometimento com a tarefa terapêutica. Os escores brutos das dimensões e o escore bruto total do WAI-S-O são obtidos pelo somatório dos pontos classificados em uma escala de tipo Likert que varia de 1 a 7, de acordo com a intensidade da afirmativa. Vale ressaltar que o instrumento não conta com estudos que apresentem normas interpretativas, bem como pontos de corte que classifiquem diferentes níveis da aliança terapêutica. Contudo, considera-se que escores elevados nas respectivas dimensões que o compõem indicam uma melhor qualidade da aliança terapêutica.

\section{Procedimento}

Avaliou-se um processo completo de Psicoterapia Breve Psicodinâmica (PBP) de um indivíduo adulto atendido em clínica-escola e que foi registrado em vídeo mediante autorização formal prévia por meio do preenchimento do Termo de Consentimento Livre e Esclarecido por ambos os participantes da pesquisa: paciente e psicoterapeuta. O projeto de pesquisa foi aprovado pelo Comitê de Ética em Pesquisa (CEP) da universidade em que foi realizada a pesquisa, CEP $N^{\circ} 1165 / 09 / 2009$ e Certificado de Apresentação para Apreciação Ética (CAAE) $\mathrm{N}^{\circ}$ 0063.0.272.000-09. Também foi obtida autorização dos responsáveis pela instituição para utilização do material a ser analisado. As sessões foram transcritas na íntegra e procurou-se seguir os padrões apresentados por Mergenthaler e Stinton (1992) para material de psicoterapia visando manter a fidelidade ao texto e preservando ao máximo a entonação e inflexões do sujeito para garantir acesso ao conjunto do material para as avaliações propostas.

O processo foi dividido em fase inicial, medial e final. As três primeiras sessões correspondem à primeira fase, de avaliação inicial. Nela, há o estabelecimento de um enquadre terapêutico, além de os esforços do psicoterapeuta serem 
direcionados para o entendimento da dinâmica do funcionamento do paciente. A fase medial, ou desenrolar do processo, engloba da $4^{\mathrm{a}}$ até a $7^{\mathrm{a}}$ sessão. Esta etapa é marcada pelo trabalho focalizado e pela atividade mais ou menos diretiva do psicoterapeuta, de acordo com a estratégia interventiva adotada por ele. Ainda, busca-se manter uma aliança positiva atrelada à forte motivação para mudança. $\mathrm{Na}$ fase final, que compreende da $8^{\mathrm{a}}$ até a $11^{\mathrm{a}}$ sessão, confere-se importância ao trabalho de término, no qual há a preocupação em retomar as experiências que o paciente obteve durante o processo, permitindo que ele incorpore os ganhos obtidos e adquira maior autonomia na resolução de dificuldades apresentadas inicialmente (Yoshida \& Enéas, 2013). A partir das transcrições, as intervenções da psicoterapeuta foram classificadas em todas as sessões e a aliança terapêutica avaliada nas sessões 3,7 e 10, cumprindo especificações metodológicas das diferentes etapas do processo.
Todas as análises foram antecedidas de consenso entre quatro juízes independentes, membros do grupo de pesquisa, familiarizados com os instrumentos utilizados: três doutores em psicologia, com 35, 34 e 15 anos de experiência clínica respectivamente, e um aluno de doutorado em psicologia, com cinco anos de experiência clínica. Calculado acordo simples entre eles, obteve-se $80 \%$ na análise das intervenções terapêuticas e $100 \%$ para análise da Aliança Terapêutica.

\section{Resultados}

Foram consideradas as intervenções verbais da psicoterapeuta, de natureza expressiva e suportiva, durante cada uma das sessões. As intervenções verbais do psicoterapeuta estão apresentadas na Tabela 1 , com as frequências de cada intervenção e o número total de intervenções realizadas por sessão.

Tabela 1

Distribuição da Natureza das Intervenções nas Diferentes Fases do Processo e das Frequências Absolutas das Intervenções Expressivas e Suportivas em Todas as Sessões do Processo

\begin{tabular}{|c|c|c|c|c|c|c|c|c|c|c|c|c|}
\hline \multirow[t]{2}{*}{ Fase } & \multicolumn{2}{|c|}{ Natureza das Intervenções } & \multirow[t]{2}{*}{ Sessão } & \multicolumn{8}{|c|}{ Intervenções Realizadas } & \multirow[t]{2}{*}{ Total } \\
\hline & Expressiva & Suportiva & & 1 & 2 & 3 & 4 & 6 & 7 & 8 & 11 & \\
\hline & & & 1 & & 1 & 7 & 5 & 15 & 5 & & 5 & 38 \\
\hline \multirow[t]{3}{*}{ Inicial } & $62,5 \%$ & $37,5 \%$ & 2 & 2 & 21 & 4 & 7 & 17 & 15 & 1 & 3 & 70 \\
\hline & & & 3 & 1 & 15 & 8 & 9 & 24 & 14 & 2 & 2 & 75 \\
\hline & & & 4 & 2 & 10 & 3 & 9 & 33 & 10 & 3 & 12 & 82 \\
\hline \multirow[t]{4}{*}{ Medial } & $57,6 \%$ & $42,4 \%$ & 5 & 1 & 14 & 3 & 15 & 24 & 11 & 1 & 4 & 73 \\
\hline & & & 6 & 2 & 15 & 12 & 7 & 17 & 19 & 7 & 7 & 86 \\
\hline & & & 7 & 2 & 15 & 7 & 25 & 21 & 7 & 5 & 5 & 87 \\
\hline & & & 8 & 1 & 10 & 7 & & 26 & 18 & 23 & 20 & 105 \\
\hline \multirow[t]{3}{*}{ Final } & $50,7 \%$ & $49,3 \%$ & 9 & 2 & 18 & 7 & 14 & 16 & 5 & 14 & 4 & 80 \\
\hline & & & 10 & & 22 & 10 & 14 & 25 & 9 & 2 & 3 & 85 \\
\hline & & & 11 & 1 & 16 & 18 & 9 & 25 & 20 & 20 & 20 & 129 \\
\hline Total & & & & 14 & 157 & 86 & 114 & 243 & 133 & 78 & 85 & 910 \\
\hline
\end{tabular}

Notas. Intervenções realizadas: 1. Interpretação, 2. Confrontação, 3. Clarificação, 4. Assinalamento, 6. Validação empática 7. Recapitulação, 8. Conselhos e elogios, 11. Fornecer informações. 
A Tabela 1 apresenta a distribuição quanto à natureza das intervenções realizadas em cada fase do processo. Observa-se a predominância de intervenções Expressivas na fase Inicial e na Medial. Já na fase Final há quase uma equiparação entre as intervenções de natureza Expressiva e Suportiva. Na fase inicial do processo, o número de intervenções foi menor que nas outras etapas (fase medial e final). Houve predomínio de intervenções expressivas, mais especificamente do tipo Confrontação $(n=37)$ e Recapitulação $(n=34)$. Nota-se também a elevada frequência da intervenção Validação Empática $(n=56)$, de natureza suportiva. Na segunda etapa do pro- cesso, a intervenção de natureza expressiva do tipo Confrontação continua sendo predominante $(n=54)$ e houve um aumento no tipo Assinalamento $(n=56)$. No que diz respeito às intervenções de natureza suportiva, o tipo Validação Empática foi a mais preeminente $(n=95)$. Por fim, a fase final foi marcada pelo elevado número de intervenções expressivas do tipo Confrontação $(n=66)$, Clarificação $(n=42)$, Assinalamento $(n=37)$ e Recapitulação $(n=52)$ e também pelo número significativo de todas as intervenções suportivas, a saber, Validação empática $(n=92)$, Conselhos e elogios $(n=59)$ e Fornecer informações $(n=47)$.

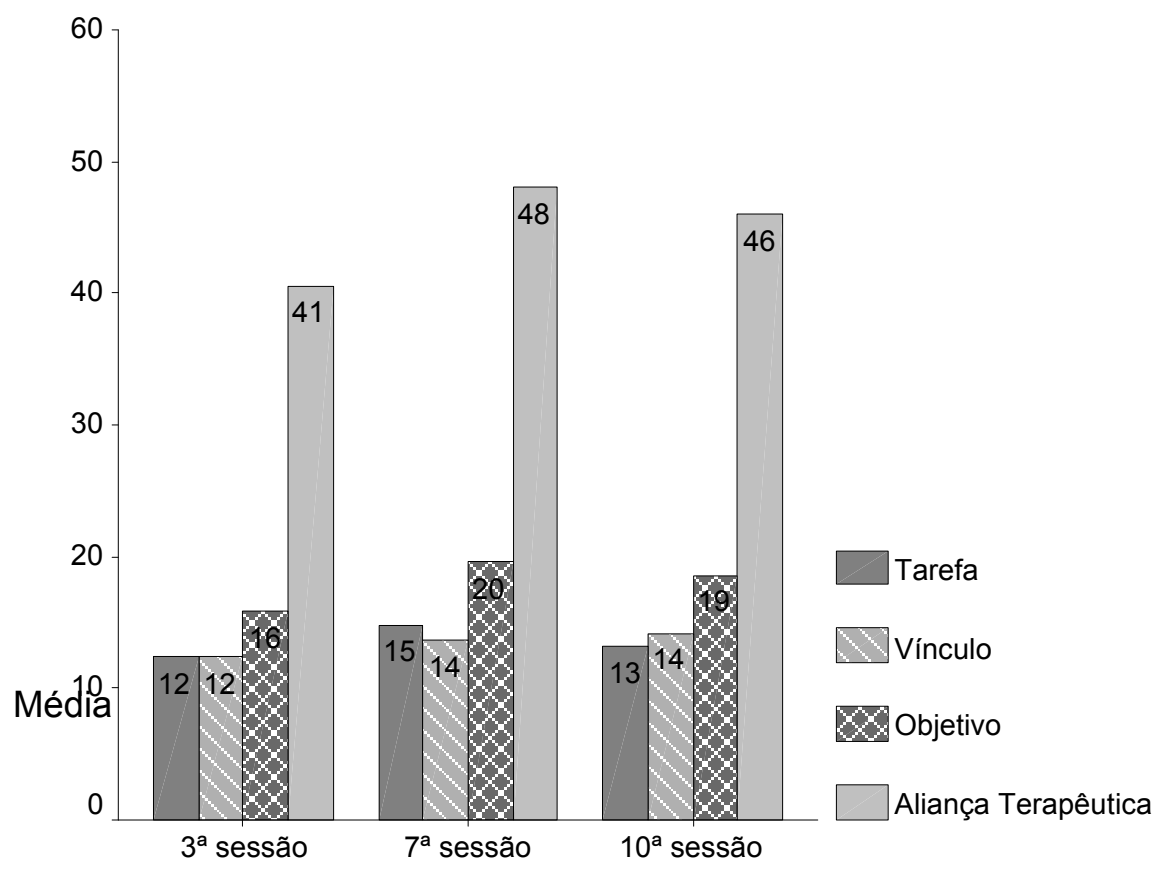

Figura 1. Escore médio da Aliança terapêutica e suas dimensões.

Já a Figura 1 apresenta os escores médios obtidos para aliança terapêutica e suas respectivas dimensões nas diferentes etapas do processo, observando-se a evolução da qualidade da aliança terapêutica entre a terceira (41) e sétima sessão (48), e pequeno retrocesso quando comparada à décima sessão (46) quando avaliado o escore total. Já em relação às diferentes dimensões da aliança terapêutica: Tarefa, Vínculo e Objetivo, observa-se o mesmo movimento, aumento dos escores apresentados entre a terceira $(12 / 12 / 16)$ e sétima sessão (15/14/20) acompa- nhados de ligeira diminuição na décima sessão $(13 / 14 / 19)$ respectivamente.

\section{Discussão}

No tocante às intervenções, tanto a primeira como a etapa medial do processo, foram marcadas pela maior frequência de intervenções expressivas $(62,5 \%$ e $57,6 \%$, respectivamente), e houve um equilíbrio entre os dois tipos na fase final (50,7\% e 49,3\%). Na mesma direção, Yoshida et al. (2009) encontraram, no estudo 
de um processo de psicoterapia breve psicodinâmica de paciente adulto, uma postura bastante expressiva por parte da psicoterapeuta, já na segunda sessão. Esta postura foi mantida na fase medial. Na fase final foi encontrada uma postura complementar por parte da psicoterapeuta, oferecendo apoio e suporte assim como utilizando intervenções expressivas. Despland et al. (2001) apontaram que as intervenções suportivas não são suficientes para a construção de uma boa aliança terapêutica e que um leque de intervenções expressivas, específicas para cada nível do funcionamento defensivo do paciente são necessárias para otimizar o crescimento da aliança.

Apesar de a fase inicial ter sido predominantemente expressiva, um olhar atento à primeira sessão vê destacada a Validação empática $(n=15)$ como a intervenção mais frequente. Isto pode indicar que esta sessão teve como objetivo inicial estabelecer uma aliança de trabalho positiva além de realizar uma tarefa inerente aos processos breves, na qual o psicoterapeuta busca ativamente colher informações a respeito do paciente (Simon, 1989; Yoshida \& Enéas, 2013; Younes, Lessa, Yamamoto, Coniaric, \& Ditzz, 2010). Conforme a Figura 1, a aliança terapêutica ainda não está totalmente consolidada nessa fase e o aumento da intervenção de tipo Validação empática ao longo da segunda $(n=17) \mathrm{e}$ da terceira $(n=24)$ sessão, sugere a intenção de fortalecê-la e, deste modo, possibilitar que a paciente aceite as intervenções de natureza Expressiva (Luborsky, 1984). No entanto, já na segunda e na terceira sessões, as intervenções do tipo Clarificação e Recapitulação tomaram lugar de destaque, indicando que a psicoterapeuta tentava resumir os pontos essenciais desta etapa de avaliação, na qual devem ter sido evidenciados aspectos que antes a paciente evitava.

$\mathrm{Na}$ fase medial, as intervenções expressivas foram prevalentes, especialmente as do tipo Confrontação $(n=54)$, que tiveram crescimento nessa fase do processo, e a ocorrência de Interpretação $(n=7)$ em todas as sessões. Supõe-se que essas intervenções foram utilizadas com intuito de ampliar a consciência da paciente, através da identificação de seus conflitos inconscientes e da demonstração das relações entre suas difi- culdades atuais, expressas em sua queixa, e seus conflitos de ordens psicológicas, que se estabeleceram no decorrer de seu desenvolvimento. Ainda no que respeita à fase medial, é notável o alto número da intervenção Validação Empática $(n=95)$, empregada em todas as sessões, associada a outras intervenções suportivas. Observa-se que a aliança terapêutica já está consolidada nessa etapa e a sintonia entre paciente e psicoterapeuta, observada nos tipos de intervenções, se dá provavelmente pela preocupação da segunda em continuar a fortalecer o vínculo já estabelecido na fase inicial do processo. Isso vai ao encontro das expectativas teóricas, de que um bom vínculo estabelecido entre ambos pode gerar uma relação de confiança e compromisso mútuo, que contribui positivamente para desenvolvimento e sucesso do tratamento (Constantino, 2012; Santibáñez Fernandez et al., 2008).

Em relação à última fase do processo, observa-se que a psicoterapia foi adquirindo um aspecto mais suportivo, chegando ao equilíbrio entre os dois polos. Uma possibilidade é de que, na fase final da psicoterapia, houve maior expressão e comunicação sobre o conflito central de relacionamento da paciente (medo de ser abandonada) e, por isso, tenha sido necessária especial atenção ao processo de separação e de término do processo psicoterápico. Esses aspectos podem também ter se refletido no ligeiro decréscimo na aliança terapêutica, uma vez que nesta fase do processo costumam ser reativados os conflitos inconscientes relativos aos sintomas, como, por exemplo, a vivência fantasiosa de abandono dos pais. A explicitação deste conteúdo exige do psicoterapeuta, não só intervenções de natureza expressiva, mas também, uma sólida base suportiva, para que o paciente aceite tais formulações e associe tais conflitos infantis às dificuldades transferenciais (Enéas \& Rocha, 2011).

Outro ponto a ser destacado é o aumento na utilização da intervenção expressiva do tipo Recapitulação $(n=20)$ na $11^{\circ}$ sessão, assim como o aumento de duas intervenções de natureza suportiva, até o momento pouco utilizadas, como Conselho e elogios $(n=20)$ e Fornecer informação $(n=20)$. É possível inferir que a psicoterapeuta procurou ajudá-la na superação das ansiedades 
levantadas no processo de término e, neste sentido, pode-se afirmar que o desfecho satisfatório desta etapa do processo dependerá da elaboração de um importante conflito da paciente (Coelho-Filho, 1997; Luborsky, 1984; Yoshida \& Enéas, 2013). Estes achados corroboram a perspectivas de que qualquer psicoterapia só pode ser considerada acabada após o rebaixamento das ansiedades levantadas nessa etapa do processo (Enéas \& Rocha, 2011).

Ressalta-se ainda que em relação à aliança terapêutica, mais especificamente na dimensão "objetivo", houve predomínio de escores mais elevados nas diferentes fases avaliadas, provavelmente porque se tratou de $\mathrm{PB}$, modalidade na qual os objetivos do processo são retomados do começo ao fim através de procedimentos como planejamento terapêutico, revisão de meio de processo e trabalho de término (Enéas \& Rocha, 2011; Yoshida \& Enéas, 2013). O que vai ao encontro das hipóteses levantadas por Horvath (1994) de que a aliança terapêutica nas fases iniciais da terapia seria em grande parte indiferenciada e global, enquanto no decorrer do tratamento a importância de cada uma de suas dimensões se distinguiria em decorrência do modelo de psicoterápico proposto.

\section{Considerações Finais}

Uma vez que o objetivo deste trabalho não foi o de estabelecer relações de causa e efeito entre as intervenções terapêuticas em cada fase do processo e a qualidade da aliança terapêutica, pode-se perceber que a natureza das intervenções e a aliança terapêutica modularam conforme as características inerentes a cada fase do processo, em especial a etapa de término (Krause et al., 2007; Krause et al., 2006). A maior expressividade, nas fases inicial e medial, pode dever-se à postura ativa da psicoterapeuta (Yoshida \& Enéas, 2013) buscando tornar conscientes os aspectos inconscientes associados à queixa apresentada pela paciente. Já na fase de término, nota-se a considerável diminuição na utilização das intervenções de natureza expressiva, uma vez que é possível a reedição do conflito relacional da paciente em sua relação com a terapeuta, exigindo da última também um trabalho suportivo. Nessa direção, Enéas e Dantas (2011), ao se basearem na obra de Kernberg, afirmaram que as psicoterapias expressivas contribuem fortemente para elaboração de conflitos, bem como para fortalecimento egoico dos pacientes.

Além de as modulações na aliança terapêutica e das intervenções poderem ter modulado segundo as tarefas e objetivos das diferentes etapas da psicoterapia, a aliança terapêutica pode ter modulado segundo as intervenções da terapeuta. Neste sentido, Gelso (2015) afirma que se o relacionamento real entre terapeuta e paciente é a base do relacionamento como um todo, então a aliança de trabalho (o autor prefere esta denominação) é o que mais diretamente possibilita que o trabalho psicoterapêutico seja feito, assim sendo, as intervenções são escolhidas também a fim de garantir que a qualidade da aliança não seja perdida e os resultados possam ser alcançados.

Este trabalho tem limitações e uma a ser destacada consiste no fato de que as análises foram planejadas a posteriori da realização do processo psicoterapêutico, o que impossibilitou a triangulação das avaliações e a resposta a medidas de autorrelato tanto pela paciente quanto pela psicoterapeuta. A realização de estudos de caso por meio de métodos qualitativos e quantitativos, por exemplo, envolvendo a avaliação de resultados, pode auxiliar no fortalecimento dos resultados encontrados. Yoshida (2008) considerou que, em estudos de caso, a significância clínica da mudança em psicoterapia pode ser determinada por medidas de autorrelato. Desta feita, algumas questões podem ser levantadas, como por exemplo, o impacto do tipo de intervenção sobre o processo de mudança do paciente; o peso das condições do paciente (diagnóstico, expectativas acerca da psicoterapia, para citar algumas) sobre o desenvolvimento da aliança terapêutica e sobre o curso da psicoterapia.

\section{Referências}

Bernecker, S. L., Levy, K. N., \& Ellison, J. D. (2014). A meta-analysis of the relation between patient adult attachment style and the working alliance. Psychotherapy Research, 24(1), 12-24. doi:10.1 080/10503307.2013.809561 
Busseri, M. A., \& Tyler, J. D. (2003). Interchangeability of the Working Alliance Inventory and Working Alliance Inventory, Short Form. Psychological Assessment, 15(2), 193-197. doi:10.1037/1040-3590.15.2.193

Coelho-Filho, J. G. (1997). Término em psicoterapia dinâmica breve. In C. D. Segre (Ed.), Psicoterapia breve. São Paulo, SP: Lemos.

Constantino, M. (2012). Believing is seeing: An envolving research program on patients' psychotherapy expectations. Psychotherapy Research, 22(2), 127-138. doi:10.1080/10503307.2012.66 3512

Del Re, A. C., Flückiger, C., Horvath, A. O., Symonds, D., \& Wampold, B. E. (2012). Therapist effects in the therapeutic alliance-outcome relationship: A restricted-maximum likelihood meta-analysis. Clinical Psychology Review, 32(7), 642-649. doi:10.1016/j.cpr.2012.07.002

Despland, J. N., Roten, Y., Despars, J., Stigler, M., \& Perry, J. C. (2001). Contribution of patient defense mechanisms and therapist interventions to the development of early therapeutic alliance in a Brief Psychodynamic Investigation. Journal of Psychotherapy Practice and Research, 10(3), 155-164.

Eells, T. D. (2007). Generating and generalizing knowledge about psychotherapy from pragmatic case studies. Pragmatic Case Studies in Psychotherapy, 3(1), 35-54. doi:10.14713/pcsp. v3i1.893

Enéas, M. L. E., \& Dantas, M. S. (2011). Critérios de indicação de psicoterapia breve em clínica-escola com pacientes difíceis. In S. F. S. Cavalini \& C. Batista (Eds.), Clínica psicodinâmica: Olhares contemporâneos (pp. 153-170). São Paulo, SP: Vetor.

Enéas, M. L. E., \& Rocha, G. M. A. (2011). Momentos decisivos em psicoterapia breve: Manejo do término. In S. F. S. Cavalini \& C. Batista (Eds.), Clínica psicodinâmica: Olhares contemporâneos (pp. 129-144). São Paulo, SP: Vetor.

Falkenström, F., Granström, F., \& Homqvist, R. (2014). Working alliance predicts psychohterapy outcome even while controlling for prior symptom improvement. Psychotherapy Research, 24(2), 146-159. doi:10.1080/10503307.2013 .847985

Fiorini, H. J. (2004). Teoria e técnica de psicoterapias. São Paulo, SP: Martins Fontes.
Gabbard, G. O. (2006). Psiquiatria psicodinâmica na prática clínica (4. ed.). Porto Alegre, RS: Artes Médicas.

Gelso, C. (2015). A tripartite model of the therapeutic relationship: Theory, research and practice. Psychotherapy Research, 24(2), 117-131. doi:10.10 80/10503307.2013.845920

Horvath, A. O (1994). Empirical validation of Bordin's Phantheoretical Model of the Alliance: The Working Alliance Inventory Perspective. In A. O. Horvath \& L. S. Greenberg (Eds.), The Working Alliance: Theory, research, and practice (pp. 1-9). New York: John Wiley \& Sons.

Horvath, A. O., \& Greenberg, L. S. (1989). Development and validation of the Working Alliance Inventory. Journal of Counseling Psychology, $36(2), 223-233$.

Khater, E., Peixoto, E. M., Honda, G. C., Enéas, M. L. E., \& Yoshida, E. M. P. (2014). Momentos chave e natureza das intervenções do terapeuta em Psicoterapia Breve Psicodinâmica. Psico-USF, 19(2), 233-242. doi:10.1590/141382712014019002010

Krause, M., de la Parra, G., Arístegui, R., Dagnino, P., Tomicic, A., Valdés, N., ...Ben-Dov, P. (2007). The evolution of therapeutic change studied through generic change indicators. Psychotherapy Research, 17(6), 673-689. doi:10.1080/10503300601158814

Krause, M., de la Parra, G., Arístegui, R., Dagnino, P., Tomicic, A., Valdés, N., ...Ramírez, I. (2006). Indicadores genéricos de cambio en el proceso psicoterapéutico. Revista Latinoamericana de Psicología, 38(2), 299-325.

Lhullier, A. C., Nunes, M. L. T., \& Horta, B. L. (2006). Preditores de abandono de psicoterapia em pacientes de clínica-escola. In E. F. de M. Silvares (Ed.), Atendimento psicológico em clínicas-escola (pp. 229-256). Campinas, SP: Alínea.

Luborsky, L. (1984). Principles of psychoanalytic psychotherapy: A manual for supportive-expressive treatment. New York: Basic Books.

Luborsky, L. (2000). A pattern-setting therapeutic alliance study revisited. Psychotherapy Research, 10(1), 17-29. doi:10.1080/713663591

Machado, P. P., \& Horvath, A. (1999). Inventário da Aliança Terapêutica: Versão portuguesa do Working Alliance Inventory. In M. R. Simões, 
L. S. Almeida, \& M. Gonçalves (Eds.), Provas psicológicas em Portugal (Vol. 2). Braga, Portugal: Sistemas Humanos e Organizacionais.

Martin, D. J., Garske, J. P., \& Davis, M. K. (2000). Relation of the therapeutic alliance with outcome and other variables: A meta-analytic review. Journal of Consulting and Clinical Psychology, 68, 438-450. doi:10.1037/0022-006X.68.3.438

Mergenthaler, E., \& Stinton, C. H. (1992). Psychotherapy transcription standards._Psychotherapy Research, 2, 125-142.

Meyer, S. B. (2006). Metodologia de Pesquisa da Psicoterapia em Clínicas-Escola. In E. F. de M. Silvares (Ed.), Atendimento psicológico em clínicas-escola (pp. 23-41). Campinas, SP: Alínea.

Muran, J. C., \& Barber, J. P. (2010). The therapeutic alliance - An evidence-based guide to practice. New York: Guilford Press.

Prado, O. Z., \& Meyer, S. B. (2004). Relação terapêutica: A perspectiva comportamental. Evidências e o Inventário de Aliança de Trabalho (WAI). Revista Brasileira de Terapia Comportamental e Cognitiva, 6(2), 201-209.

Prochaska, J. O. (1995). An ecletic and integrative approach: Transtheoretical therapy. In A. S. Gurman \& S. B. Messer (Eds.), Essencial Psychotherapies: Theory and practice (pp. 403440). New York: Guilford.

Safran, J., Muran, J. C., Demaria, A., Boutwell, C., Eubanks-Carter, C., \& Winston, A. (2014). Investigating the impact of alliance focused training on interpersonal process and therapists' capacity for experiential reflection. Psychotherapy Research, 24(3), 269-285. doi:10.1080/1050330 7.2013.874054

Santibáñez Fernandez, P. M., Román Mella, M. F., Lucero Chenevard, C., Espinoza García, A. E., Irribarra Cáceres, D. E., \& Müller Vergara, P. A. (2008). Variables inespecíficas en Psicoterapia. Terapia Psicológica, 26, 89-98. doi:10.4067/ S0718-48082008000100008

Simon, R. (1989). Psicologia Clínica Preventiva: Novos fundamentos. São Paulo, SP: Editora Pedagógica e Universitária.

Slonim, D. A., Shefler, G., Gvirsman, S. D., \& Tishby, O. (2011). Changes in rigidity and symptoms among adolescents in psychodynamic psychotherapy. Psychotherapy Research, 21(6), 685697. doi:10.1080/10503307.2011.602753
Tishby, O., \& Vered, M. (2011). Countertransference in the treatment of adolescents and its manifestation in the therapist-patient relationship. Psychotherapy Research, 21(6), 621-630. doi:10.1080/ 10503307.2011.598579

Tracey, T. J., \& Kokotovic, A. M. (1989). Factor structure of the Working Alliance Inventory. Psychological Assessment: A Journal of Consulting and Clinical Psychology, (3), 207-210.

Wiseman, H., \& Tishby, O. (2014). The therapeutic relationship: Multiple lenses and innovations. Introduction to a special section. Psychotherapy Research, 24(3), 251-256. doi:10.1080/1050330 7.2014.892648

Yoshida, E. M. P. (2008). Significância clínica de mudança em processo de psicoterapia psicodinâmica breve. Paideia (Ribeirão Preto), 18(40), 305316. doi:10.1590/S0103-863X2008000200008

Yoshida, E. M. P. (2012). Psicoterapias psicodinâmicas. In M. E. N. Lipp \& E. M. P. Yoshida (Eds.), Psicoterapias breves nos diferentes estágios evolutivos (pp. 1-17). São Paulo, SP: Casa do Psicólogo.

Yoshida, E. M. P., \& Enéas, M. L. E. (2013). A proposta do Núcleo de Estudos e Pesquisa em Psicoterapia Breve para Adultos. In E. M. P. Yoshida \& M. L. E. Enéas (Eds.), Psicoterapias psicodinâmicas breves: Propostas atuais (3. ed., pp. 231-269). Campinas, SP: Alínea.

Yoshida, E. M. P., Elyseu, S., Jr., Silva, F. R. C. S., Finotelli, I., Jr., Sanches, F. M., Penteado, E. F., ...Enéas, M. L. E. (2009). Psicoterapia psicodinâmica breve: Estratégia terapêutica e mudança no padrão de relacionamento conflituoso. Psico-USF, 14(3), 275-285. doi:10.1590/S141382712009000300004

Yoshida, E. M. P., Gatti, A. L., Enéas, M. L. E., \& Coelho-Filho, J. G. (1997). Aliança Terapêutica, Transferência e Motivação num processo de Psicoterapia Breve. Mudanças, 7, 141-154.

Younes, J. A., Lessa, F., Yamamoto, K., Coniaric, J., \& Ditzz, M. (2010). Psicoterapia Breve Operacionalizada e crise por expectativa de perda: Um estudo de caso. Psicologia Argumento, 28(63), 303-309.

Recebido: 10/02/2015

$1^{a}$ revisão: 18/08/2015

$2^{a}$ revisão: 14/09/2015

Aceite final: 12/10/2015 